\title{
A case of refractory Hashimoto's encephalopathy demonstrating improvement with plasmapheresis
}

\author{
Sarah E. Nelson ${ }^{1}$, Yasir N. Jassam ${ }^{1}$, Lynne P. Taylor ${ }^{2}$ \\ 1. Neurology, Tufts Medical Center, Boston, United States. 2. Hematology-Oncology, Tufts Medical Center, Boston, United \\ States.
}

Correspondence: Sarah Nelson. Address: 20 2nd Street, Unit H321, Cambridge, MA 02141, United States. Email: snelson@tuftsmedicalcenter.org

Received: February 27, 2014

Accepted: April 2, 2014

Online Published: April 10, 2014

DOI : $10.5430 /$ crim.v1n2p83

URL: http://dx.doi.org/10.5430/crim.v1n2p83

\begin{abstract}
Hashimoto's encephalopathy (HE) can present with multiple neurologic manifestations and various neuroimaging findings. While its progressive form is often well-recognized, patients with a waxing and waning course may provide diagnostic difficulty. Treatment with corticosteroids has been well-documented, but the benefit of immune-related therapies such as plasmapheresis has not been solidified. Here we describe a patient with waxing and waning signs and symptoms as well as significant fluctuations in neuroimaging findings that initially caused uncertainty regarding her diagnosis. After several largely negative tests including two brain biopsies, she was found to have high levels of anti-thyroid antibodies. Though sometimes found in disorders such as Hashimoto's thyroiditis and Graves' disease, these laboratory values in the setting of unremarkable thyroid function tests and her symptoms and their initial responsiveness to corticosteroids helped solidify her diagnosis as HE. However, due to refractory symptoms and signs, this patient required plasmapheresis. We demonstrate clinical, serological, and radiological improvement with this yet unconfirmed therapy, thus raising questions about the appropriateness of the term "steroid-responsive encephalopathy".
\end{abstract}

\section{Keywords}

Hashimoto disease, Autoimmune thyroiditis, Neuroimaging, Cognition disorders, Aphasia

\section{I ntroduction}

Hashimoto's thyroiditis was first described by Hakaru Hashimoto in 1912 but it was Lord Brain who initially noted the association between thyroiditis and an unusual encephalopathy in 1966 that he called Hashimoto's encephalopathy ${ }^{[1-2]}$. It is unclear if the anti-thyroid antibodies that characterize this disorder are an autoimmune epiphenomenon or are pathogenic ${ }^{[1]}$. Many prefer the term "steroid responsive encephalopathy associated with autoimmune thyroiditis" because of this condition's responsiveness to corticosteroids - greater than $90 \%$ of patients in some reviews ${ }^{[3,4]}$. HE can present with several neurological manifestations and disease courses that can cause initial diagnostic confusion. Neuroimaging is often normal. We present a 74-year-old female with no personal or family history of autoimmune disease who presented with waxing and waning neurological symptoms and striking imaging findings for nearly a year before ultimately being diagnosed with HE. Her very thorough evaluation included multiple laboratory tests and studies in addition to two brain biopsies. In addition, we also demonstrate efficacy of plasmapheresis in our patient. 


\section{Case presentation}

A 74-year-old Russian female with a history of hypertension, coronary artery disease, diabetes mellitus, and treated hypothyroidism and a family history remarkable only for cardiovascular disorders presented in April 2012 with several months of confusion and aphasia. MRI brain demonstrated cortical T2 hyperintense lesions in multiple vascular territories. On further workup, MRA head and neck was unremarkable and transesophageal echocardiogram demonstrated an ejection fraction of 55\% with no thrombus or interatrial shunt. With the conclusion that the patient had likely suffered from a cardioembolic stroke, she was placed on dabigatran. The patient was doing well when 10 days prior to her admission in May 2012 she became increasingly confused, stopped speaking English, and had difficulty understanding language.

Though recurrent stroke was considered, a broad differential was initially entertained, including metabolic and infectious etiologies. However, initial negative serum studies included basic metabolic profile, liver function tests, renal function tests, coagulation values, thyroid stimulating hormone (TSH), thyrotropin binding inhibitory immunoglobulin, and vitamin B12 level. Complete blood count and urine and blood cultures were also unremarkable. For concern of a connective tissue disorder or malignancy as an etiology, C-reactive protein and erythrocyte sedimentation rate were tested and were unremarkable. In addition, CT chest, abdomen, and pelvis with and without contrast was unremarkable. Human immunodeficiency (HIV) 1 and 2 antibody and rapid plasma reagin were also tested given that HIV and syphilis can sometimes cause altered mental status, but these values were also unremarkable. Given the appearance of her previous MRI findings, Lyme antibody and angiotensin converting enzyme were sent but were normal.

Furthermore, given the above unremarkable work-up, the patient underwent a lumbar puncture. However, negative cerebrospinal fluid (CSF) studies included oligoclonal bands, IgG index, myelin basic protein, herpes simplex virus 1 and 2, and 14-3-3 protein. CSF protein was slightly elevated at $58 \mathrm{mg} / \mathrm{dl}$ (normal 15-45 mg/dl), but CSF glucose and white blood cell count were unremarkable. An electroencephalogram (EEG) performed for concern of seizures showed only moderate cerebral dysfunction with possible right temporal/posterior cortical irritability.

With the above studies largely unrevealing, HE was considered and anti-thyroid antibodies were then sent. These results showed thyroperoxidase (TPO) microsomal antibody $73 \mathrm{U} / \mathrm{mL}$ (normal 0-5.9 U/mL) and thyroglobulin antibody 636.1 $\mathrm{U} / \mathrm{mL}$ (normal 0-9.9 U/mL). Notably, in addition to an unremarkable TSH (3.21 $\mu \mathrm{IU} / \mathrm{mL}$ (normal 0.35-5.50 $\mu \mathrm{IU} / \mathrm{mL}$ )), other thyroid function tests were total T3 $62 \mathrm{ng} / \mathrm{dl}$ (normal 60-181 ng/dl), free T4 1.35 ng/dl (normal 0.80-1.80 ng/dl), and total T4 $8.7 \mu \mathrm{g} / \mathrm{dl}$ (normal 4.5-10.9 ug/dl). Her elevated anti-thyroid antibodies in the setting of her clinical presentation and normal thyroid function tests suggested that the patient was euthyroid.

MRI brain showed multifocal areas of abnormal T2/FLAIR hyperintense signal involving the cortex and subcortical white matter in bilateral frontal, temporal, occipital, and parietal lobes some of which were increased or decreased in size or new with no enhancement noted (see Figure 1). To further clarify her diagnosis, she underwent a right temporal brain biopsy that showed marked reactive gliosis and rare lymphocytes with no evidence of encephalitis, lymphoma, or prion diseases.

Despite this nonspecific biopsy result, the patient was treated empirically with corticosteroids for a presumed diagnosis of HE or central nervous system (CNS) lymphoma, thought possibly to have been missed on the biopsy. One month later, while still on corticosteroids, the patient was greatly improved. She was better able to converse in both English and Russian though was occasionally nonfluent and was independent in her activities of daily living.

A follow-up MRI brain in July 2012 continued to show multifocal areas of abnormal T2/FLAIR hyperintensity involving the cortex and subcortical white matter with some lesions smaller and others new or increased in size. In an outpatient visit that month, her family noted that the patient's cognitive status was improving, including her attention, but was not yet at baseline. She was able to perform the cognitive tasks she had previously completed, asked good questions, and better engaged in Russian. Repeat thyroglobulin antibody at that time was $403.0 \mathrm{U} / \mathrm{mL}$. Due to her improving neurological examination she was weaned off corticosteroids. 


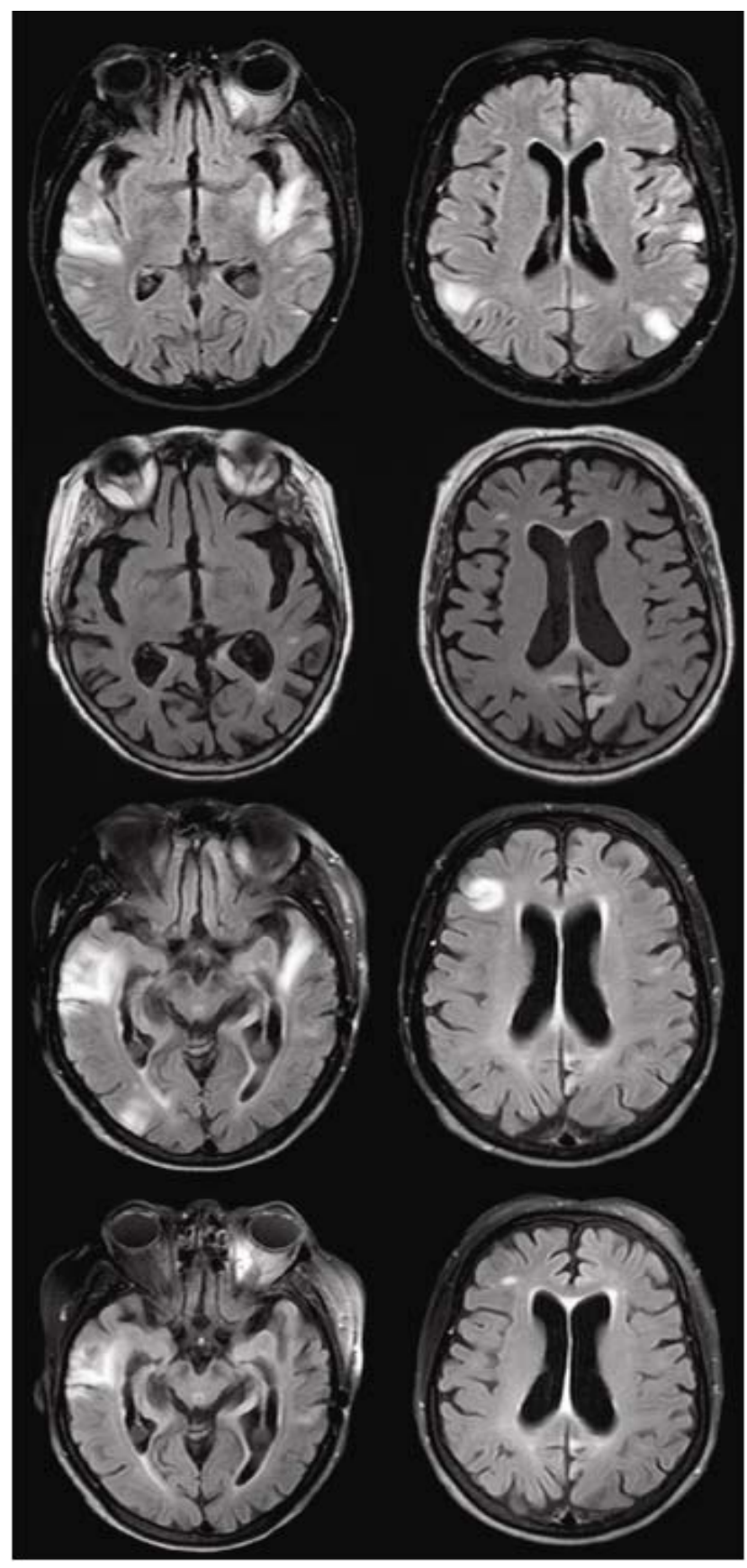

Figure 1. Axial FLAIR MRI brain over time. (Top row) Different axial slices from May 2012 demonstrating multifocal T2/FLAIR hyperintensities involving the cortex and subcortical white matter. (Second row) Similar axial slices as above from March 2013 demonstrating improvement in the multifocal T2/FLAIR hyperintensities. (Third row) Similar axial slices as above from June 2013 showing increased leukoencephalopathy. (Bottom row) Similar axial slices as above from August 2013 showing improved leukoencephalopathy.

In late November 2012, however, the patient experienced increased confusion, and a repeat thyroglobulin antibody level in early December 2012 was $>1000.0 \mathrm{U} / \mathrm{mL}$. A repeat brain biopsy - completed in an attempt to confirm a diagnosis of presumed $\mathrm{HE}$ - was performed in the left temporal region and was again nonspecific in demonstrating moderate to marked gliosis and no evidence for a lymphoproliferative disorder. 
Despite the nondiagnostic second brain biopsy, she was diagnosed with HE due to her neurological symptoms, elevated anti-thyroid antibodies, response to corticosteroids, and relatively unremarkable supporting studies. She was restarted and improved on corticosteroids. MRI brain from March 2013 demonstrated marked improvement in the T2/FLAIR hyperintensities (see Figure 1).

Unfortunately, however, the patient's symptoms continued to fluctuate. In May 2013 while still on corticosteroids, she presented with worsening aphasia and executive dysfunction including attentional deficits. Thyroglobulin antibody was 704.4 U/mL, and MRI brain showed new lesions. Her corticosteroid dose was increased, and azathioprine was started for chronic immunosuppressive therapy.

Nonetheless, deteriorating language and memory problems were noted in June 2013. MRI brain demonstrated increased foci of T2/FLAIR hyperintensities (see Figure 1). Due to the refractory nature of her symptoms despite treatment with corticosteroids and azathioprine, she was started on plasmapheresis. Thus far she has undergone plasmapheresis approximately every 2-3 days between June 28-July 8, 2013, October 8-21, 2013, and January 23-31, 2014.

Since undergoing two courses of plasmapheresis to date, the patient has improved clinically: she has not experienced confusion, her language is without paraphrasic errors, and she maintains household activities. Interestingly, her anti-thyroid antibody levels decreased after at least two of her courses of plasmapheresis (antibody levels were not measured after her October 2013 plasmapheresis) (see Table 1). In addition, her neuroimaging findings have also improved since starting plasmaphereis (see Figure 1). She is currently maintained on corticosteroids, azathioprine, and plasmapheresis.

Table 1. Clinical Correlation with Anti-Thyroid Antibody Levels

\begin{tabular}{lccc}
\hline Date & $\begin{array}{c}\text { TPO Microsomal Antibody } \\
\mathbf{( 0 . 0 - 5 . 9 ~} \mathbf{~ / m )})\end{array}$ & $\begin{array}{c}\text { Thyroglobulin Antibody } \\
\mathbf{( 0 . 0 - 9 . 9 ~ U / m L )}\end{array}$ & Clinical Course \\
\hline May 2012 & 73.0 & 636.1 & Initial presentation \\
July 2012 & $\mathrm{NM}$ & 403.0 & Improved \\
December 2012 & $\mathrm{NM}$ & $>1000.0$ & Worsened \\
March 2013 & $\mathrm{NM}$ & $\mathrm{NM}$ & Improved \\
May 2013 & 134.8 & 704.4 & Worsened \\
June 2013 & 103.2 & 432 & Worsened \\
August 2013 & 60.6 & $\mathrm{NM}$ & Improved \\
October 2013 & 52.9 & $\mathrm{NM}$ & Stable \\
January 22, 2014 & 122.7 & $\mathrm{NM}$ & Worsened \\
January 31, 2014 & 51.6 & $\mathrm{NM}$ & Improved \\
\hline
\end{tabular}

Note: Abbreviations: $\mathrm{TPO}=$ thyroid peroxidase, $\mathrm{NM}=$ not measured. Normal values are provided in parentheses

\section{Discussion}

While Hakaru Hashimoto first described Hashimoto's thyroiditis in 1912, the disorder now known as HE - also known as Hashimoto's encephalitis, steroid-responsive encephalopathy associated with autoimmune thyroiditis, and nonvasculitic autoimmune inflammatory meningoencephalitis - was first noted in 1966 by Lord Brain, who described what is considered the first case of this disorder ${ }^{[1,2]}$.

As demonstrated in our patient, multiple neurologic abnormalities can occur in patients with HE, including encephalopathy, stroke-like episodes, psychosis, visual hallucinations, aphasia, ataxia, seizures, myoclonus, and tremor. Supportive laboratory tests include thyroid-related antibodies such as anti-thyrotropin, anti-TPO, anti-thyroglobulin, and 
anti-alpha-enolase. An association with thyroid disorders is sometimes found but neurologic symptoms do not appear to depend on thyroid status ${ }^{[3,4]}$. Though anti-alpha-enolase was not tested in our patient, her anti-thyroid antibodies were noted to be significantly elevated. As discussed above, diagnoses such as Hashimoto's thyroiditis and Graves' disease can be considered in patients with elevated anti-thyroid antibodies but this was considered unlikely in our patient given her normal thyroid function tests and clinical exam.

In the CSF the most frequent finding is elevated protein, as found in our patient, though anti-thyroid antibodies have also been noted (these were not tested in our patient) ${ }^{[1,3]}$. EEG abnormalities are seen in a majority of patients, most commonly diffuse slowing but also triphasic waves, periodic sharp waves, and frontal rhythmic slowing ${ }^{[1,4,5]}$. There are no neuropathology findings specific to $\mathrm{HE}$ though an encephalitic or vasculitic process has been debated in the literature ${ }^{[1,3,4,6,7]}$. A relationship to CNS demyelination has also been shown ${ }^{[4,8,9]}$. Our patient's brain biopsies and EEG were overall considered nonspecific.

The diagnosis of HE requires the presence of anti-thyroid antibodies, a progressive or relapsing-remitting course, and the exclusion of other etiologies ${ }^{[4,10]}$. In 1999, Peschen-Rosin et al proposed the first set of diagnostic criteria: episodes of relapsing myoclonus, focal neurological deficits, seizures, or psychiatric disorders in addition to at least three of the following: heterogeneous but unrevealing MRI brain, abnormal EEG, high anti-thyroid antibody levels, elevated protein and/or oligoclonal bands in the CSF, and good response to steroids ${ }^{[11]}$.

MRI brain findings are variable and can be normal or include focal or confluent white matter abnormalities, atrophy, and other cortex irregularities ${ }^{[1]}$. MRI abnormalities may improve with steroid treatment and can correlate with anti-thyroid antibody levels; in our patient it appears that her neuroimaging findings roughly improved along with her anti-thyroid antibody levels (see Table 1 and Figure 1 ) $^{[1]}$. MRI findings can also change over time within the same patient ${ }^{[1,4]}$. A review of $85 \mathrm{HE}$ patients showed that 42 of 82 patients (52\%) who had undergone neuroimaging had normal findings ${ }^{[3]}$. In a review of 121 patients, 71 patients were noted to have normal neuroimaging (it is unclear how many of these 121 patients underwent imaging, however) ${ }^{[4]}$. Those with abnormal scans most commonly exhibited cortical atrophy and nonspecific subcortical focal white matter abnormalities ${ }^{[3,4]}$. Thus while neuroimaging in HE is often normal, our patient demonstrated the more typical HE imaging findings. Even so, imaging in our case fluctuated dramatically over time.

Anti-thyroid antibodies is one of the signature features of HE, though as mentioned above there is uncertainty as to whether they are pathogenic or merely an autoimmune epiphenomenon ${ }^{[1]}$. While they do not necessarily parallel the clinical status of HE patients, exceptions have been noted ${ }^{[3,4,12]}$. Our patient is remarkable in that symptom improvement and worsening has roughly correlated with anti-thyroid antibody levels decreasing and increasing, respectively (see Table 1).

The mean age of patients with HE was approximately mid-40-years-old in two reviews. Though our case could be considered unusual given that she initially presented at 74-years-old, a large age range in which this disorder can manifest has been found (9- to 78-years-old in one review, 8- to 86-years-old in another review) ${ }^{[3,4]}$.

Corticosteroids have been the mainstay of therapy for HE, and greater than $90 \%$ of patients improved on this therapy in two large reviews of HE patients ${ }^{[3,4]}$. However, other treatments that can be beneficial in immune-mediated disorders, such as plasmapheresis and intravenous immunoglobulin (IVIG), have been used. In particular, the data on plasmapheresis is suggestive of a possible benefit but is not conclusive. Immuosuppressant medications have also been tried including azathioprine and cyclophosphamide ${ }^{[1,3,4]}$. Our patient initially benefited from steroids, and the effects of azathioprine likely remain to be seen. Significantly, however, our patient demonstrated clinical, serological, and radiological improvement with plasmapheresis.

Our patient experienced a relapsing-remitting form of HE that was also difficult to treat. Various clinical courses can be seen in HE including progressive, relapsing-remitting, and stroke-like episodes though the progressive form may be the 
most recognizable ${ }^{[1,4]}$. In one review of $121 \mathrm{HE}$ patients, 55 (45\%) patients had relapsing courses and 10 (8\%) were treatment-refractory, including some who did not respond to more than one therapy like our patient ${ }^{[4]}$. In another study including $85 \mathrm{HE}$ patients, 51 (60\%) patients suffered a relapsing-remitting course and 8 were refractory to treatments including glucocorticoids, glucocorticoids plus levothyroxine, levothyroxine, and IVIG ${ }^{[3]}$.

We present a case of relapsing-remitting and treatment-refractory HE who, because of her varied neurological manifestations and neuroimaging findings over time, was initially challenging to diagnose. Ultimately, her positive anti-thyroid antibodies, symptomatic response to corticosteroids, and exclusion of other etiologies left little doubt of her diagnosis of HE. Our case is also unusual because significant fluctuations in her neuroimaging findings occurred over time. Correlation between clinical status and anti-thyroid antibody levels in HE is not always seen but was generally true in our patient. Finally, our demonstration of plasmapheresis efficacy adds to the small body of literature regarding the use of this therapy in HE patients. Physicians should consider the diagnosis of HE in patients presenting with relapsing-remitting symptoms and imaging that may be initially suggestive of other diagnoses.

\section{References}

[1] Schiess N, Pardo C. Hashimoto’s encephalopathy. Ann NY Acad Sci. 2008; 1142: 254-265. http://dx.doi.org/10.1196/annals.1444.018

[2] Brain L, Jellinek EH, Ball K. Hashimoto’s disease and encephalopathy. Lancet. 1966; 2(7462): 512-514. http://dx.doi.org/10.1016/S0140-6736(66)92876-5

[3] Chong JY, Rowland LP, Utiger RD. Hashimoto encephalopathy: syndrome or myth? Arch Neurol. 2003; 60: 164-171. http://dx.doi.org/10.1001/archneur.60.2.164

[4] Ferracci F, Carnevale A. The neurological disorder associated with thyroid autoimmunity. J Neurol. 2006; 253: $975-984$. http://dx.doi.org/10.1007/s00415-006-0170-7

[5] Henchey R, Cibula J, Helveston W, et al. Electroencephalographic findings in Hashimoto’s encephalopathy. Neurology. 1995; 45: 977-981. http://dx.doi.org/10.1212/WNL.45.5.977

[6] Doherty CP, Schlossmacher M, Torres N, et al. Hashimoto’s encephalopathy mimicking Creutzfeldt-Jakob disease: brain biopsy findings. J Neurol Neurosurg Psychiatry. 2002; 73: 601-602. http://dx.doi.org/10.1136/jnnp.73.5.601-a

[7] Paulus W, Nolte KW. Neuropathology of Hashimoto’s encephalopathy. J Neurol Neurosurg Psychiatry. 2003; $74: 1009$. http://dx.doi.org/10.1136/jnnp.74.7.1009

[8] Chaudhuri A, Behan PO. Hashimoto's encephalopathy: a relapsing form of acute disseminated encephalomyelitis. J Neurol Sci. 2005; 235: 75-76. http://dx.doi.org/10.1016/j.jns.2005.04.007

[9] Mahad DJ, Staugaitis S, Ruggieri P, et al. Steroid-responsive encephalopathy associated with autoimmune thyroiditis and primary CNS demyelination. J Neurol Sci. 2005; 228: 3-5. http://dx.doi.org/10.1016/j.jns.2004.08.015

[10] Mijajlovic M, Mikovic M, Dackovic J, et al. Clinical manifestations, diagnostic criteria and therapy of Hashimoto’s encephalopathy: report of two cases. J Neurol Sci. 2010; 288: 194-196. http://dx.doi.org/10.1016/j.jns.2009.09.030

[11] Peschen-Rosin R, Schabet M, Dichgans J. Manifestation of Hashimoto’s encephalopathy years before onset of thyroid disease. Eur Neurol. 1999; 41: 79-84. http://dx.doi.org/10.1159/000008007

[12] Yuceyar N, Karadeniz M, Erdogan M, et al. Thyrotoxic autoimmune encephalopathy in a female patient: only partial response to typical immunosuppressant treatment and remission after thyroidectomy. Clin Neurol Neurosurg. 2007; 109: 458-462. http://dx.doi.org/10.1016/j.clineuro.2007.02.006 